\title{
Optimal Supplier Choice with Discounting
}

\author{
B. Goldengorin, J. Keane, V. Kuzmenko \& M. K-S. Tso
}

First version: 24 September 2008

Research Report No. 12, 2008, Probability and Statistics Group

School of Mathematics, The University of Manchester 


\title{
Optimal supplier choice with discounting
}

\author{
B Goldengorin, J. Keane, V Kuzmenko and MK-S Tso* \\ * corresponding author
}

Keywords: Allocation, decision support systems, integer programming, location

\begin{abstract}
This paper investigates a model for pricing the demand for a set of goods when suppliers operate discount schedules based on total business value. We formulate the buyers's decision problem as a mixed binary integer program (MIP) which is a generalization of the capacitated facility location problem (CFLP). A branch and bound procedure using Lagrangean relaxation and subgradient optimization is developed for solving large-scale problems that can arise when suppliers' discount schedules contain multiple price breaks. Results of computer trials on specially adapted large benchmark instances of the CFLP, confirm that a subgradient optimization procedure based on Shor and Zhurbenko's r-algorithm, which employs a space dilation strategy in the direction of the difference between two successive subgradients, can solve such instances efficiently.
\end{abstract}

\section{Introduction}

The rapid development of business-to-business (B2B) electronic markets has trigggered the need for efficient algorithms to allow an internet agent to source, i.e. to price in real time, an order for goods from multiple competing suppliers. The task is often complicated by two factors: 1) fixed costs which serve to reduce the number of suppliers actually used to fulfill an order, and 2) discounts schedules offered by suppliers to encourage purchase of greater quantities. Such discount schedules may involve either the cancellation of a fixed charges, e.g. to cover carriage costs, or increasing percentage reductions off list price over a sequence of pricebreaks.

Our study is motivated by the requirements of an online supplier of pharmaceuticals to high street chemists (retail pharmacists). The company acts as an internet broker in the sense that it carries no stock but, on receiving an enquiry (tentative order) for quantities of pharmaceutical products, it polls a set of wholesalers to determine which suppliers to use taking into account all applicable discounts. It then provides in real time a price quotation in answer to the enquiry based on cost, but including a profit mark-up. The enquiry is converted to a firm order if the total price of the basket of goods is judged to 
be acceptable by the customer. Thus the broker's task is to source the order at least cost. Note that the terms "enquiry" and "order" will be regarded as synonymous below.

We assume that the company has online access to a negotiated "static" price list for each supplier, and there is no aggregation of customers' enquiries or economies achievable through "bulk buying", although in certain business contexts this would be an interesting possibility to consider.

We formulate below a general model for the "buyer's decision problem" $(B D P)$ incorporating two common types of discount offered by suppliers: Type $A$ : a cancellation or reduction in the fixed charge, and Type B: a percentage off the list price of each item. Discounts based on the value of an order have been termed a "business volume" discount (BVD) in contrast to a "total quantity" discount (TQD) when price breaks are defined by number of units purchased.(Goossens et al., 2004). Discounting may also take the form either of an "all units" policy modelled here, or an "incremental" policy. For a perspective on discounting theory and practice, see (Munson and Rosenblatt, 1998) where a field study on 39 firms is reported. An optimization model for vendor selection in the presence of price breaks was reported in (Chaudhry et al., 1993), though at that time there was no requirement for an online tool. The precise form of discounting employed will depend on the application, however the methods we develop for $B V D$ can be be easily generalized to other cases.

We show that the optimal allocation of a basket of goods to a set of suppliers in the market is an integer programming problem which resembles the capacitated facility location problem (CFLP). A supplier's price schedule represents a set of per unit costs and fixed setup costs. An interesting feature of our model is that the cost functions implied by the discount structures described here are distinctive, being discontinous and in general neither concave nor convex. We note the resemblance to "staircase cost functions" proposed for modelling production and distribution costs when a plant can be constructed in a range of sizes on a single site incurring scale-dependent setup (and running) costs (Holmberg, 1994), (Holmberg and Ling, 1997). Unlike our situation however, their fixed costs increase monotonically with plant capacity.

The fast response time required in an online context motivates the need for an efficient computational procedure for solving $B D P$. Whilst the efficient solution of large scale instances of CFLP has been the subject of much research in over many years (Cornuejols et al., 1991), (Agar and Salhi, 1998), (Bornstein and Azlan, 1998), the development of more efficient solution heuristics for large scale problems remains an area of active research, see for example (Barahona and Chudak, 2005) and (Klose and Goertz, 2007). Such research has focussed almost exclusively on Lagrangean relaxation techniques, see (Krarup 
and Bilde, 1977),(Beasley, 1988) and (Körkel, 1989). In this paper we report the results of investigating several new heuristics for finding tight lower bounds for the Lagrangean dual problem for $B D P$ involving subgradient optimization. A novel feature of our study is the use of the "r-algorithm" proposed by Shor and Zhurbenko (Shor, 1998), (Shor and Zhurbenko, 1971) which employs space dilation techniques to implement the subgradient optimization. We note the recent development of memoryless space dilation techniques in (Sherali et al., 2001) and related work by (Wu et al., 2006) in the context of CFLP with general setup costs

An outline of our paper follows. We first give some motivating examples to further illustrate the practical context of our study. We then formulate the buyer's decision problem (BDP) as a mixed integer linear program assuming an all units $B V D$ discount policy. We show that a transformation making use of "pseudosuppliers" results in a variant of the capacitated facility location problem (CFLP) which may be solved for large scale instances (involving many suppliers and multiple price breakpoints) by an efficient Lagrangean heuristic, Shor's r-algorithm. Such a procedure which employs the geometric concept of space dilation in the direction of two successive subgradients, represents an alternative to the "classical" subgradient approach. We present the results of computer experiments on a number of generated instances (a) to compare solution efficiencies of the r-algorithm with a classical subgradient method, and (b) to examine the time requirements of solution procedures under different levels and types of discounting. We conclude with a discussion and suggestions for future research.

\section{Motivating examples}

\section{Example 1}

A mobile phone company announces that customers making over $£ 30$ of calls in a month will receive a refund of $£ 5$. In addition, customers making over $£ 50$ of calls in a month will qualify for a $20 \%$ discount from published tariffs. The discount schedule contains three piecewise linear segments created from two price breakpoints at $£ 30$ and $£ 50$, representing "all units" discounts of Type $A$ and Type $B$ respectively. The price $p(v)$ of calls made to a total value $v$ is given by

$$
p(v)= \begin{cases}v & \text { if } v<30 \\ v-5 & \text { if } 30 \leq v<50 \\ 0.8 v-5 & \text { if } 50 \leq v\end{cases}
$$


and the graph given by the bold line in Figure 1 has the typical sawtooth form (Sadrian and Yoon, 1994). From this graph we also observe the "more for less" phenomenon (Goossens et al., 2004) that it can be cheaper to make more calls if the value of telephone business is just less than either breakpoint, in order to benefit from the next discount regime. By contrast the dotted graph in Figure 1 represents the price-value relationship under an "incremental" discount scheme in which only calls made above the $£ 50$ breakpoint qualify for the reduced tariff.

\section{Example 2}

Prices of boxes of tulips and roses from two florists for are given in Table 1. Delivery charges are $£ 10$ for florist 1 and $£ 5$ for florist 2 . Charges are waived on orders over $£ 50$ in value. Optimal transportation matrices for a) 7 boxes of tulips and 3 boxes of roses, and b) 7 boxes of tulips only are given in Table 2 .

In the optimal solution to a) roses are purchased from both florists, showing that single sourcing is sub-optimal. In the optimal solution to b) it is optimal to exceed demand by ordering an additional single rose from the first florist illustrating the "more for less" phenomenon.

\section{The buyer's decision problem}

Consider a set of $n$ products (items) indexed by $j \in J$ and suppose that each item $j$ can be supplied by a common set of suppliers $s \in S$ at unit $\operatorname{cost} c_{j}^{s}$ from supplier $s$. We refer to $c^{s}=\left(c_{1}^{s}, \ldots, c_{n}^{s}\right)$ as the list price for items supplied by supplier $s$. Assume that each supplier offers discounts based on the total value of the order placed with that supplier computed according to the supplier's list price. The amount and nature of the discount is governed by a sequence of $q$ price bands. If we suppose, for ease of notation, that $q$ is the same for each supplier, then given a set of breakpoints $V_{0}^{s}<V_{1}^{s}<\ldots<V_{q}^{s}$ the price bands for supplier $s$ are consecutive intervals $I_{1}^{s}=\left[V_{0}^{s}, V_{1}^{s}\right), \ldots, I_{q}^{s}=\left[V_{q-1}^{s}, V_{q}^{s}\right)$. We will assume without loss of generality that $V_{0}^{s}=0$ and $V_{q}^{s}=\infty$. An order placed with supplier $s$ for $x_{j}^{s}$ units of item $j(j \in S)$ forms a vector $x^{s}=\left(x_{1}^{s}, \ldots, x_{n}^{s}\right)$ and has total business value $(T B V)$ given by $V^{s}\left(x^{s}\right)=\sum_{j \in J} c_{j}^{s} x_{j}^{s}$.

The discounted price $\pi^{s}\left(x^{s}\right)$ that supplier $s$ charges for the order is assumed to take the form

$$
\begin{aligned}
\pi^{s}\left(x^{s}\right) & =f_{k}^{s}+\rho_{k}^{s} \sum_{j \in J} c_{j}^{s} x_{j}^{s} \quad \text { if } V^{s}\left(x^{s}\right) \in I_{k}^{s} \\
& =f_{k}^{s}+\sum_{j \in J} c_{k j}^{s} x_{j}^{s} \quad \text { say. }
\end{aligned}
$$


for given constants $\left\{\rho_{k}^{s}\right\},\left\{f_{k}^{s}\right\}, k \in K=\{1, \ldots, q\}$. We usually expect that higher order values will attract greater levels of discount so that $1=\rho_{1}^{s} \geq \rho_{2}^{s} \geq \ldots$ and $f_{1}^{s} \geq f_{2}^{s} \geq \ldots$ Note that $1=\rho_{1}^{s}$ means that $c_{j}^{s}=c_{1 j}^{s}$, i.e. the first price band $k=1$ corresponds to list price.

The broker receives an order comprising a set of demands for $D_{j}$ units of item $j(j \in J)$ and seeks to reallocate $d=\left(D_{1}, \ldots, D_{n}\right)$ amongst the suppliers at minimum cost. The set of (sub)orders $\left\{x^{s}\right\}$ should meet the total demand

$$
\sum_{s \in S} x^{s} \geq d
$$

minimize the total cost of supply

$$
C(x)=\sum_{s \in S} \pi^{s}\left(x^{s}\right)
$$

by taking advantage of all suppliers' discounts. Minimizing (2) will of course tend to restrict the number of suppliers. We refer to this cost optimization as the buyer's decision problem (BDP) and note that $x^{s}$ may be real or integer, depending on application context.

Let $x_{k j}^{s}$ denote the quantity of item $j$ ordered from supplier $s$ in price band $k$. Now $V^{s}\left(x^{s}\right)=\sum_{j \in J} c_{j}^{s} x_{j}^{s} \in I_{k}^{s}$ for some $k=k_{s}$, say, and clearly $x_{k j}^{s}=0$ whenever $k \neq k_{s}$, since it will be optimal only for one price band ever to be used for any supplier. Define

$$
y_{k}^{s}=\left\{\begin{array}{cc}
1 & \text { if } V^{s}\left(x^{s}\right) \in I_{k}^{s} \\
0 & \text { otherwise }
\end{array}\right.
$$

and re-index

$$
y_{1}^{1}, \ldots, y_{q}^{1} ; y_{1}^{2}, \ldots, y_{q}^{2} ; \ldots ; y_{1}^{s}, \ldots, y_{q}^{s}
$$

as a single sequence $\left\{y_{i}\right\}_{i \in I}$. We henceforth refer to $I=\{1, \ldots, m\}$ as a set of pseudosuppliers. A corresponding re-indexing of $f_{k}^{s}=f_{i}, c_{k j}^{s}=c_{i j}, x_{k j}^{s}=x_{i j}$ and $I_{k}^{s}=\left[L_{i}, U_{i}\right)$ leads to the following generalized capacitated facility location problem (CFLP) in which the capacity constraints represent lower and upper bounds on $T B V$. 


$$
\begin{array}{cll}
\text { BDP: } \quad \min _{y_{i}, x_{i j}} \sum_{i \in I}\left\{f_{i} y_{i}+\sum_{j \in J} c_{i j} x_{i j}\right\} & \\
\text { s.t. } \quad L_{i} y_{i} \leq \sum_{j \in J} c_{i j}^{0} x_{i j} \leq U_{i} y_{i} & \forall i \in I, \\
& \sum_{i \in P^{s}} y_{i} \leq 1 & \forall s \in S, \\
& \sum_{i \in I} x_{i j} \geq D_{j} & \forall j \in J, \\
y_{i} \in\{0,1\} & \forall i \in I \\
x_{i j} \in \mathbb{Z}^{+} & \forall i \in I, j \in J
\end{array}
$$

where

$P^{s}$ is the set of pseudosuppliers $i$ corresponding to real supplier $s$, and

$c_{i j}^{0}=c_{1 j}^{s}$ is the unit list price of item $j$ for pseudosupplier $i$ for $i \in P^{s}$.

The objective function (4) is precisely that of a standard (either simple or capacitated) plant location model. (5) states that the $T B V$ of the suborder supplied from pseudosupplier $i$ should fall within the appropriate price band. Note that in contrast to the usual CFLP model (5) contains both lower and upper bounds. Constraint (6) ensures that at most one price list per supplier can appear in an optimal solution. The demand constraints (7) may be expressed either as equalities or as lower bounds. Our use of inequalities corresponds to the "more-for-less" formulation mentioned by Goossens et.al., (Goossens et al., 2004) Section 4.2) allowing for the possibility that it may be cheaper to overfulfill demand in order to benefit from a higher level of discount.

In practice there may be stock limits that restricted availability of some product $j$ from some supplier $s$. There may also be commercial reasons why a supplier $s$ may wish to limit or deny availability of some product $j$ within a price band $k$. We therefore include in our formulation of BDP the upper bound constraints

$$
0 \leq x_{i j} \leq S_{i j}, \quad i \in P^{s}
$$

for some constants $\left\{S_{i j}\right\}$. Finally we note that while $x_{i j}$ are integer variables in this formulation of BDP, in other applications $x_{i j}$ may be continuous, so that (9) will be replaced by $x_{i j} \in \mathbb{R}^{+}$. 


\section{Lagrangean duality}

Since no optimal solution can employ more than one price band from a single supplier, we can technically remove the upper bounds in (5) and in fact all the constraints (6) from the formulation of BDP and any optimal solution to $\mathrm{BDP}$ will remain optimal under the new formulation. However retaining the additional constraints allows tighter dual bounds in the relaxations we describe below.

We write the constraints (7) in the form $D_{j}-\sum_{i \in I} x_{i j} \leq 0$ and introduce as a vector of corresponding Lagrange multipliers, the dual variables $\lambda=$ $\left(\lambda_{1}, \ldots, \lambda_{n}\right)$ where $\lambda_{j} \geq 0, j \in J$. The Lagrangean dual problem (LDP) corresponding to BDP can be stated as

$$
\text { LDP : } \quad \max _{\lambda_{j} \geq 0} F(\lambda)
$$

where

$$
F(\lambda)=\min _{y_{i}, x_{i j}}\left\{\sum_{i=1}^{m} f_{i} y_{i}+\sum_{i=1}^{m} \sum_{j=1}^{n}\left(c_{i j}-\lambda_{j}\right) x_{i j}+\sum_{j=1}^{n} D_{j} \lambda_{j}\right\}
$$

subject to (5),(6),(8),(9), and (10). For each $\lambda \geq 0$ this Lagrangean dual subproblem is a relaxation of BDP, and as is well-known, $F(\lambda)$ is a concave piecewise-linear function. This allows us to employ subgradient techniques to seek a constrained maximum of (11) which provides the best lower bound for problem BDP.

\section{The Lagrangean heuristic}

At any node of the branch and bound tree we will have partitioned $I$ into the index sets $K_{0}, K_{1}, K_{2}$ such that

$$
y_{i}=0, \quad i \in K_{0}, \quad y_{i}=1, \quad i \in K_{1}, \quad y_{i} \in\{0,1\}, \quad i \in K_{2}
$$

Thus $K_{0}, K_{1}$ are the pseudosuppliers fixed closed, open respectively; $K_{2}$ are the undetermined pseudosuppliers. Letting $P_{L}=\left|K_{1}\right|$ and $P_{U}=\left|K_{1} \cup K_{2}\right|$ we may add the explicit bounds

$$
P_{L} \leq \sum_{i=1}^{m} y_{i} \leq P_{U}
$$

on the total number of actual suppliers used.

The solution to the Lagrangean dual subproblem (12)-(14) for a prescribed vector of Lagrange multipliers $\lambda$ can be reduced to solving two knapsack prob- 
lems. The first knapsack problem is defined for each non-closed pseudosupplier $i \in K_{1} \cup K_{2}$ and determines the contribution to the dual function (12) from pseudosupplier $i$ if open :

$$
\alpha_{i}(\lambda)=f_{i}+\min _{\left\{x_{i j}\right\}} \sum_{j=1}^{n}\left(c_{i j}-\lambda_{j}\right) x_{i j}
$$

subject to (5) and (10) where $x_{i j} \in \mathbb{Z}^{+}$. We note that solving the 1-D integer knapsack is NP-hard (Martello and Toth, 1990). However, since our aim in solving the dual subproblem is to find a lower bound to BDP we may solve instead the continous knapsack relaxation. The minimum of (15) subject to (5) and (10) over $x_{i j} \in \mathbb{R}^{+}$is achieved by a greedy heuristic, in which we order the $x_{i j}$ by non-decreasing value of the ratio $\left(c_{i j}-\lambda_{j}\right) / c_{i j}^{0}$ and set the components of $x_{i j}^{*}$ in turn to their maximum value subject to (5) and (10). In case of infeasibility, we set $y_{i}=0$ and $x_{i j}=0$ for each $j$. The speed and simplicity of this solution to the continuous relaxation is of course at the expense of an increased duality gap for $x_{i j} \in \mathbb{R}$ compared to that for $x_{i j} \in \mathbb{Z}$.

The second knapsack problem is a minimization problem on the set of Boolean variables $\left\{y_{i}\right\}$

$$
F(\lambda)=\min _{\left\{y_{i}\right\}} \sum_{i \in K_{2}} \alpha_{i}(\lambda) y_{i}+\sum_{i \in K_{1}} \alpha_{i}(\lambda)+\sum_{j=1}^{n} D_{j} \lambda_{j}
$$

subject to (6) and (8). For each supplier $s$, let $\beta_{s}=\min _{i \in P^{s} \cap K_{2}}\left\{\alpha_{i}(\lambda)\right\}$ and form the corresponding list of pseudosuppliers $i_{1}, i_{2}, \ldots$ in non-decreasing order of $\beta_{s}$. Define the sequence of partial sums $\left\{S_{t}\right\}$ by

$$
\begin{aligned}
S_{0} & =\sum_{i \in K_{1}} \alpha_{i}(\lambda)+\sum_{j=1}^{n} D_{j} \lambda_{j} \\
S_{1} & =S_{0}+\alpha_{i_{1}}(\lambda) \\
\vdots & \\
S_{t} & =S_{t-1}+\alpha_{i_{t}}(\lambda)
\end{aligned}
$$

The smallest value of $S_{t^{*}}$ such that $P_{L} \leq t^{*} \leq P_{U}$ provides an optimal solution $y^{*}$ to (16) and hence the solution to (12) subject to the given constraints. Let $Z_{U}$ be the value of the incumbent i.e. of the best feasible solution found so far. We decide the branch is fathomed if $S_{t^{*}} \geq Z_{U}$, otherwise we continue to develop this node. 


\section{Reduction tests}

This straightforward procedure for solving the dual subproblem by solving two knapsack problems provides the opportunity to check whether each $y_{i}$ variable can be fixed at 0 or 1 in subsequent branchings. Beasley develops Lagrangean heuristics for location problems in (Beasley, 1993) making extensive use of such rules first proposed in (Akinc and Khumawala, 1977). We have developed the following rules for the BDP:

Let $M^{*}$ denote the set of pseudosuppliers that have $y_{i}^{*}=1$ in the solution to the Lagrangean subproblem (12) - (14).

\section{Close penalties}

For $i \in K_{2} \cap M^{*}$ so $y_{i}^{*}=1$, consider the change in $F(\lambda)$, say $\left.\Delta F(\lambda)\right|_{y_{i}=0}$, as a result of setting $y_{i}=0$ (closing $i$ ). Let $\alpha_{\min }$ be the smallest value of $\alpha_{l}(\lambda)$ with $y_{l}^{*}=0$ such that pseudosupplier $l$ can be feasibly opened in the solution to (12), keeping all other variables unchanged. Forcing pseudosupplier $i$ closed allows $l$ to be opened if $S_{t^{*}}$ is reduced as a result. If closing $i$ causes the lower limit on cardinality of $M^{*}$ to be violated, $l$ is also forced to enter $M^{*}$. As a result of these considerations the increase in $F(\lambda)$ due to closing $i$ is

$$
\left.\Delta F(\lambda)\right|_{y_{i}=0}= \begin{cases}-\alpha_{i}(\lambda)+\alpha_{\min }, & \text { if }\left|M^{*}\right|=P_{L} \\ -\alpha_{i}(\lambda)+\min \left\{0, \alpha_{\min }\right\}, & \text { if }\left|M^{*}\right|>P_{L}\end{cases}
$$

and if $F(\lambda)+\left.\Delta F(\lambda)\right|_{y_{i}=0} \geq Z_{U}$ we discard the subproblem (12)-(14) with $y_{i}=0$ and fix $y_{i}=1$ in all subsequent completions of this branch, i.e. we transfer $i$ from $K_{2}$ to $K_{1}$.

\section{Open penalties}

For any pseudosupplier $i \in K_{2}$ satisfying $y_{i}^{*}=0$, we calculate the change $\left.\Delta F(\lambda)\right|_{y_{i}=1}$ in $F(\lambda)$ as a result of forcing $y_{i}=1$ (opening $i$ ). We need to distinguish between three cases. In the first case $i \in P_{s}$ and supplier $s$ is already represented in $M^{*}$ by some $l$ with $y_{l}^{*}=1$ (i.e. $P_{s}$ is already open in $y^{*}$ at some other level of discount). This case results in a forced exchange of $i$ with $l$. In the second case, opening pseudosupplier $i$ violates the upper limit on cardinality of $M^{*}$ so that some open pseudosupplier $l=i_{2}$, say, must be closed. In the final case, opening $i$ may allow the possibility that some supplier $i_{2}$ be closed. Let $\alpha_{\max }$ be the maximum of $\alpha_{l}(\lambda)$ over $l \in M^{*} \cap K_{2}$. In the latter two cases, the greatest reduction in $F(\lambda)$ will be achieved when $i_{2}$ is the corresponding argument to $\alpha_{\max }$. The net change in $F(\lambda)$ can be summarized 
as follows:

$$
\left.\Delta F(\lambda)\right|_{y_{i}=1}= \begin{cases}\alpha_{i}(\lambda)-\alpha_{l}(\lambda), & \text { if } P_{s} \text { is already open at } l \\ \alpha_{i}(\lambda)-\alpha_{\max }, & \text { if }\left|M^{*}\right|=P_{U} \\ \alpha_{i}(\lambda)-\max \left\{0, \alpha_{\max }\right\}, & \text { otherwise }\end{cases}
$$

\section{Narrowing the bounds $P_{L}, P_{U}$}

The sequence $\left\{S_{t}\right\}$ given by (17) has the property that $S_{t}$ is the value of the optimal dual solution $F(\lambda)$ subject to the cardinality constraint $\left|M^{*}\right|=t$. In other words, each such value denoted by $F_{t}(\lambda)$ is a lower bound for the original BDP with $\sum_{i=1}^{m} y_{i}=t$. For very little extra computation when solving the dual subproblem, it is possible to determine the maximal interval $\left[t_{1}, t_{2}\right]$ for which $F(\lambda)<Z_{U} \forall t \in\left[t_{1}, t_{2}\right]$. This allows us to reduce the interval $\left[P_{L}, P_{U}\right]$ to $\left[t_{1}, t_{2}\right]$. We note that $\left[t_{1}, t_{2}\right]$ will be non-empty since $S_{t^{*}}<Z_{U}$, otherwise this branch would have been fathomed on solving the dual subproblem.

\section{Subgradient procedure}

The family of "r-algorithms" (Shor and Stetsyuk, 2002) has been developed for the unconstrained maximization of concave objective functions over a continuous domain. The r-algorithm is a refinement of the classical subgradient method with space dilation developed by Shor and co-workers in the early 1970's (Shor, 1970) which Todd has identified as an example of a rank-one quasi-Newton method (Todd, 1986). The refinement employs space dilation in the direction of the difference between two successive subgradients. At the optimal solution $\left\{x_{i j}^{*}\right\}$ to the Lagrangean dual subproblem at iteration $k$ with $\lambda=\lambda^{k}$, the value of the supergradient is

$$
\nabla_{j} F\left(\lambda^{k}\right)=D_{j}-\sum_{i=1}^{m} x_{i j,}^{*} \quad j=1, \ldots, n
$$

The constraints $\lambda_{j} \geq 0$ can be incorporated by the use of a symmetrizing transformation $\lambda_{j}=\left|u_{i}\right|$ where $u_{i} \in \mathbb{R}$. The computational efficiency of the r-algorithm depends on a choice of the space dilation coefficient $\alpha$ and tuning the values of the step multiplier. We have applied the r-algorithm with constant coefficient $\alpha$ of space dilation in the interval $[2,4]$ and adaptive tuning of the step multiplier (see (Shor, 1998), p.104). This choice seeks to achieve as large as possible an improvement of the objective function along the current direction of search. In computer experiments reported below, timings are compared for the classical subgradient method and the r-algorithm on simulated instances. In our 
experiments, no speed improvements were introduced by the recently developed memoryless space dilation and reduction strategy of (Sherali et al., 2001).

\section{Generating feasible solutions}

The branch-and-bound algorithm requires a heuristic to generate good feasible solutions in order to prune the tree by discarding manifestly sub-optimal subproblems. Given a set of open pseudosuppliers $M^{*}$ from the solution to the Lagrangean dual problem (11) we seek

$$
Z\left(M^{*}\right)=\sum_{i \in M^{*}} f_{i}+\min _{x_{i j}} \sum_{i \in M^{*}} \sum_{j \in J} c_{i j} x_{i j}
$$

subject to

$$
\begin{array}{cc}
L_{i} \leq \sum_{j \in J} c_{i j}^{0} x_{i j} \leq U_{i}, & i \in M^{*} \\
\sum_{i \in M^{*}} x_{i j} \geq D_{j}, & j \in J
\end{array}
$$

and constraints 9 and 10. This problem, whether $x_{i j}$ is integer or real, is a generalized transportation problem with upper and lower bounds on the total value sourced from each pseudosupplier. In computer experiments, we have taken the above model with only the lower bound in (21) and used CPLEX to solve the continuous problem. The optimal solution is rounded up to the nearest integer solution. We then check whether the upper bound in (21) holds. If not, owing to the nature of our discount function we are able to find another pseudosupplier for which constraint (21) will be valid and for which the optimal value will be less.

\section{Branching procedure}

We have implemented two heuristics for choosing the branching variable from amongst the undecided variables $\left\{y_{i}\right\}_{i \in K_{2}}$. In the first approach we have used a heuristic procedure due to (Belyaeva et al., 1978) and not widely known, for estimating the optimal values of $\left\{y_{i}\right\}$ in the solution to the LP primal corresponding to the relaxed Lagrangean dual subproblem (12). The calculation is based on an estimate

$$
y_{i}^{*}=\lim _{k \rightarrow \infty}\left\{\frac{\sum_{t=1}^{k} h_{t} y_{i}^{t}}{\sum_{t=1}^{k} h_{t}}\right\}
$$

using the values of $y_{i}$ encountered during the subgradient iteration $\left\{\lambda^{t}\right\}_{t=0}^{\infty}$ which converges in the point $\lambda^{*}$. Here $h_{t}$ and and $y_{i}^{t}$ are respectively the step- 
length and value of $y_{i}$ at step $t$ of the iteration. If all $y_{i}^{*}$ are integer, then no branching is needed. Otherwise we select the "most non-integral" $y_{i}$ using the smallest value of $\left|y_{i}^{*}-\frac{1}{2}\right|$ as the criterion and branch first on the subproblem $y_{i}=1$.

In our second approach which is new, we determined the branching variable $y_{i}$ through the "Open" and "Close" penalties obtained after solving the dual subproblem. We define

$$
\begin{aligned}
& F_{i}^{O}=\max _{k}\left\{F\left(\lambda^{k}\right)+\left.\Delta F\left(\lambda^{k}\right)\right|_{y_{i}=1}\right\} \\
& F_{i}^{C}=\max _{k}\left\{F\left(\lambda^{k}\right)+\left.\Delta F\left(\lambda^{k}\right)\right|_{y_{i}=0}\right\}
\end{aligned}
$$

and branch by the variable $i$ for which $F_{i}^{C}-F_{i}^{O}$ is maximal. If $F_{i}^{C} \geq F_{i}^{O}$, then we set $y_{i}=1$ (open). Otherwise we set $y_{i}=0$ (closed). Our computational experiments on Set 3 instances below have shown that such an assignment of $y_{i}$ leads very fast to a feasible solution which is close to an optimal solution.

\section{Computational experiments}

We carried out computer experiments to compare the performance of the branch and bound procedure described above using two subgradient methods for finding lower bounds, the "r-algorithm" of Shor and the "classical" subgradient approach of Christofides and Beasley (Christofides and Beasley, 1983). In this comparison, three datasets were used. Problem sets 1 and 2 were adapted from three uncapacitated facility location (UFL) problem instances derived from the OR benchmarks library (Beasley, 1990): Cap 71, Cap 101 and Cap 131. Problem set 3 was adapted from the instance Uniform-123 in the Library of Discrete Location Problems maintained by the Sobolev Institute of Mathematics in Novosibirsk (Kochetov and Ivanenko, 2003). It is known to be a hard instance.

\section{OR Lib - Set 1}

This problem set is adapted from three instances in the OR benchmarks library: Cap 71 with $m=16$ plants, $n=50$ customers; Cap 101 with $m=25, n=50$; Cap 131 with $m=n=50$. A single breakpoint at $V=50,000$ was introduced in all suppliers, when a fixed Type $A$ discount of value 7500 became applicable. The range of variation of costs (unit prices) $\left\{c_{i j}\right\}$ is $\left[0,1.4 \times 10^{6}\right]$ that of demands $\left\{D_{j}\right\}$ is $\left[31,1.3 \times 10^{4}\right]$. We wished to compare the efficiency of the subgradient procedures and the sensitivity of each procedure to scaling of the the demands $\left\{D_{j}\right\}$. 
Table 3 summarizes the comparison. Detailed are the number of branchings, the total number of iterations and computing times for both methods (seconds on a PC with installed Intel Pentium 200 chip). Each instance was solved with and without scaling the demands $\left\{D_{j}\right\}$ to unity.

We see that the number of branchings is uniformly less for the r-algorithm, indicating that the r-algorithm returns tighter lower bounds than the classical subgradient method. Solution times however are not markedly different due to greater overheads in computing subgradients for the r-algorithm. Scaling before solution is effective for the simple subgradient method, but does not affect the performance of the r-algorithm.

\section{OR Lib - Set 2}

Table 4 presents CPU timings (s.) to solve instances from problem set 2. Each instance is derived from the OR Library dataset CAP131 with $m=n=50$ by adding different types and levels of discount to create two price bands. The level of discount offered in the second price band is indicated in the dataset name as a percentage reduction, respectively in fixed cost $\left\{f_{i}\right\}$ in the case of Type $\mathrm{A}$ and from the unit prices $\left\{c_{i j}\right\}$ in the case of Type B.

The results indicate that the r-algorithm generally takes more time to compute the bound than the classical subgradient method. The case A0+B0 is the undiscounted case which corresponds to SPLP without pseudosuppliers and is clearly very fast. Problems with Type A discounts require much more computing time to solve than instances with a purely Type B discount although the instance A100 which corresponds to the total removal of fixed costs for orders above the value threshold is also solved in less time. The most difficult cases are Type A instances with 50-60\% discount. Timings are much reduced when both types of discount are applicable. This might be explained, intuitively, by the observation that when suppliers offers both types of discount simultaneously there is an incentive to use fewer suppliers, making the corresponding instance easier to solve.

\section{Sobolev Institute - Set 3}

For problem set 3 , instances with $m=n=100$ and different types/levels of discount were generated from a single dataset Uniform-123 containing $m=100$ plants and $n=100$ customers with $D_{j}=1$, each $j \in\{1, \ldots, n\}$. Each supplier was given a single price break with $f_{1}^{s}=3000$ and $f_{2}^{s}=0$, giving rise to 200 pseudosuppliers. $D_{j}$ was either left unscaled or generated as uniformly distributed pseudorandom numbers in the range $[1, D]$ where $D$ is tabulated. 
Table 5 shows CPU times (s.) taken to find exact and approximate solutions to within a prescribed level of accuracy. The value of the best feasible solution found so far, $Z_{U}$, is also given with * indicating termination occurs at a true minimum.

We observe that the classical subgradient approach solves many of our instances more efficiently than the r-algorithm. However, solution times are generally of the same order of magnitude, although in one instance (B50;3\%) the r-algorithm took more than 50 times longer. We note, however that the performance of the r-algorithm is also influenced by different choices of tuning parameters. When scaling of $\left\{D_{j}\right\}$ is performed so the demand for different products varies, we see an increase in the solution times using the simple subgradient algorithm. However the solution times by the r-algorithm remain much more stable. The largest solution times for approximate solutions are observed on instances with the pure Type A discounts of $50 \%$.

\section{Conclusion}

We have addressed a general problem of importance in e-commerce, how to determine a minimum cost assignment of an order for a basket of goods to a set of suppliers taking into account fixed charges and some common types of discounting policies. The fast response time required in an online context motivates the need for an efficient computational procedure.

Our integer programming formulation may be regarded as a new type of capacitated facility location model for which solution procedures based on Lagrangean relaxation have been extensively studied. Efficient procedures should be capable of rapidly solving large instances with possibly scores of suppliers offering hundreds of products and operating discount schedules containing multiple breakpoints. Two branch-and-bound algorithms have been implemented which differ in the method of computing the lower bounds. The first employs the dual-based Lagrangean heuristic based on the "classical" subgradient method of Bilde and Krarup (1977) and the second is the "r-algorithm" based on space dilation in the direction of difference of two consecutive supergradients due to Shor (1970) . Details of a new branching rule and fathoming heuristics have been provided on the basis of Open and Close penalties akin to those first proposed by Khumawala (Khumawala, 1972), (Akinc and Khumawala, 1977).

Computational experiments show that

- although the r-algorthm produces tighter bounds giving a reduced branch and bound tree, the classical subgradient algorithm achieved comparable solution times on test problems. 
- the "self-tuning" nature of the r-algorithm means however that solution times may be less sensitive to large differences in scale of the problem coefficients if this method is adopted.

- discounts of Type A (change in fixed costs) may present more of a computational challenge than Type B ( $\%$ change in variable cost).

We conclude that Lagrangean relaxation techniques can efficiently solve to optimality large-scale instances of the buyer's decision problem involving many hundreds of suppliers and price lists containing multiple price breakpoints On the basis of the instances solved, it is unclear whether the extra programming required to implement the r-algorithm will be justified by faster solution speeds.

The methods developed here are the basis for further studies into online sourcing of goods with different discounting policies. The issue of bulking or aggregating orders is one we have not addressed here. In practice it is common for suppliers to operate more complex discounting policies, e.g. based on the total value of orders aggregated over a time window. Such an environment requires an optimal strategy that evolves over time. A supplier faced with current competition in a specific market for products and services may make use of these optimal solutions as a tool for evaluating alternative price lists and discounting strategies. We foresee natural developments of our model motivated by recent research into the theory and practice of reverse auctions.

Finally, we note that optimization models involving linear and fixed transaction costs have recently been proposed for portfolio optimization in the financial context. Such models incorporate a stochastic dimension as the objective function coefficients are rates of return which are assumed to occupy a probability space (Lobo et al., 2007).

\section{Acknowledgements}

A preliminary version of this paper was presented at The 3rd International Conference on Algorithmic Aspects in Information and Management (AAIM'07) held during 6-8 June 2007 in Portland, Oregon, USA.

We gratefully acknowledge the assistance provided by the Manchester Institute for the Mathematical Sciences (MIMS) in providing financial support to allow visits of the first and the third authors (BG, VK) to the University of Manchester in 2004 and 2006. 


\section{References}

Agar, M.C. and S. Salhi (1998), 'Lagrangean heuristics applied to a variety of large capacitated plant location problems.', J. Oper. Res. Soc. 49(10), 1072-1084.

Akinc, U. and B.M. Khumawala (1977), 'An efficient branch and bound algorithm for the capacitated warehouse location problem.', Manage. Sci. 23, 585-594.

Barahona, F. and F.A. Chudak (2005), 'Near-optimal solutions to large-scale facility location problems.', Discrete Optim. 2(1), 35-50.

Beasley, J.E. (1988), 'An algorithm for solving large capacitated warehouse location problems.', Eur. J. Oper. Res. 33, 314-325.

Beasley, J.E. (1990), 'OR-Library: distributing test problems by electronic mail', J. Oper. Res. Soc. 41(11), 1069-1072.

Beasley, J.E. (1993), 'Lagrangean heuristics for location problems.', Eur. J. Oper. Res. 65(3), 383-399.

Belyaeva, L.V., N.G. Zhurbenko and N.Z. Shor (1978), 'On a method of solving a class of dynamic distributive problems.', Economics and Mathematical Methods (in Russian) 14, 137-146.

Bornstein, C.T. and H.B. Azlan (1998), 'The use of reduction tests and simulated annealing for the capacitated plant location problem.', Location Science 6, 67-81.

Chaudhry, S., F. Forst and J. Zydiak (1993), 'Vendor selection with price breaks.', Eur. J. Oper. Res. 70, 52-66.

Christofides, N. and J.E. Beasley (1983), 'Extensions to a Lagrangean relaxation approach for the capacitated warehouse location problem.', Eur. J. Oper. Res. 12, 19-28.

Cornuejols, G., R. Sridharan and J.M. Thizy (1991), 'A comparison of heuristics and relaxations for the capacitated plant location problem.', Eur. J. Oper. Res. 50(3), 280-297.

Goossens, D.R., A.J.T. Maas, F.C.R. Spieksma and J.J. van der Klundert (2004), Exact algorithms for procurement problems under a total quantity discount structure., Technical Report OR 0452, Faculty of Economics and Applied Economics, K.U. Leuven. 
Holmberg, K. (1994), 'Solving the staircase cost facility location problem with decomposition and piecewise linearization.', Eur. J. Oper. Res. 75(1), 4161.

Holmberg, K. and J. Ling (1997), 'A Lagrangean heuristic for the facility location problem with staircase costs.', Eur. J. Oper. Res. 97(1), 63-74.

Khumawala, B.M. (1972), 'An efficient branch and bound algorithm for the warehouse location problem.', Manage. Sci. B 18(12), 718-731.

Klose, A. and S. Goertz (2007), 'A branch-and-price algorithm for the capacitated facility location problem.', Eur. J. Oper. Res. 179(3), 1109-1125.

Kochetov, Yu. and D. Ivanenko (2003), Computationally difficult instances for the uncapacitated facility location problem., in 'Proceedings of the Fifth Metaheuristics International Conference(MIC2003), Kyoto, Japan'.

Körkel, M. (1989), 'On the exact solution of large-scale simple plant location problems', Eur. J. Oper. Res. 39(2), 157-173.

Krarup, J. and O. Bilde (1977), Plant location, set covering and economic lot size: an $O(m n)$-algorithm for structured problems, in 'Numerische Methoden bei Optimierungsaufgaben, Band 3 (Tagung, Oberwolfach, 1976)', Birkhäuser, Basel, pp. 155-180. Internat. Ser. Numer. Math., Vol. 36.

Lobo, M.S., M. Fazel and S. Boyd (2007), 'Portfolio optimization with linear and fixed transaction costs.', Ann. Oper. Res. 152(1), 376-394.

Martello, S. and P. Toth (1990), Knapsack problems. Algorithms and computer implementations., Chichester: John Wiley \& Sons Ltd.

Munson, C.L. and M.J. Rosenblatt (1998), 'Theories and realities of quantity discounts: an exploratory study.', Prod. Oper. Manag. 7(4), 352-369.

Sadrian, A. and Y. Yoon (1994), 'A procurement decision support system in business volume discounts environments.', Oper. Res. 42(1), 14-23.

Sherali, H. D., G. Choi and Z. Ansari (2001), 'Limited memory space dilation and reduction algorithms.', Comput. Optim. Appl. 19(1), 55-77.

Shor, N.Z. (1970), 'Utilization of the operation of space dilation in the minimization of convex functions.', Cybern. Syst. Anal. 6(1), 7-15.

Shor, N.Z. (1998), Nondifferentiable optimization and polynomial problems., Nonconvex Optimization and Its Applications. 24. Dordrecht: Kluwer Academic Publishers. 
Shor, N.Z. and N.G. Zhurbenko (1971), 'A minimization method using space dilation in the direction of the difference between two successive subgradients.', Cybernetics 7(3), 450-459.

Shor, N.Z. and P.I. Stetsyuk (2002), 'Lagrangian bounds in multiextremal polynomial and discrete optimization problems.', J. Glob. Optim. 23(1), 1-41.

Todd, M.J. (1986), 'The symmetric rank-one quasi-Newton method is a spacedilation subgradient algorithm.', Oper. Res. Lett. 5, 217-219.

Wu, L.Y., X.S. Zhang and J.L. Zhang (2006), 'Capacitated facility location problem with general setup cost.', Comput. Oper. Res. 33(5), 1226-1241. 
Figure 1:

$B V D$ "sawtooth discount function

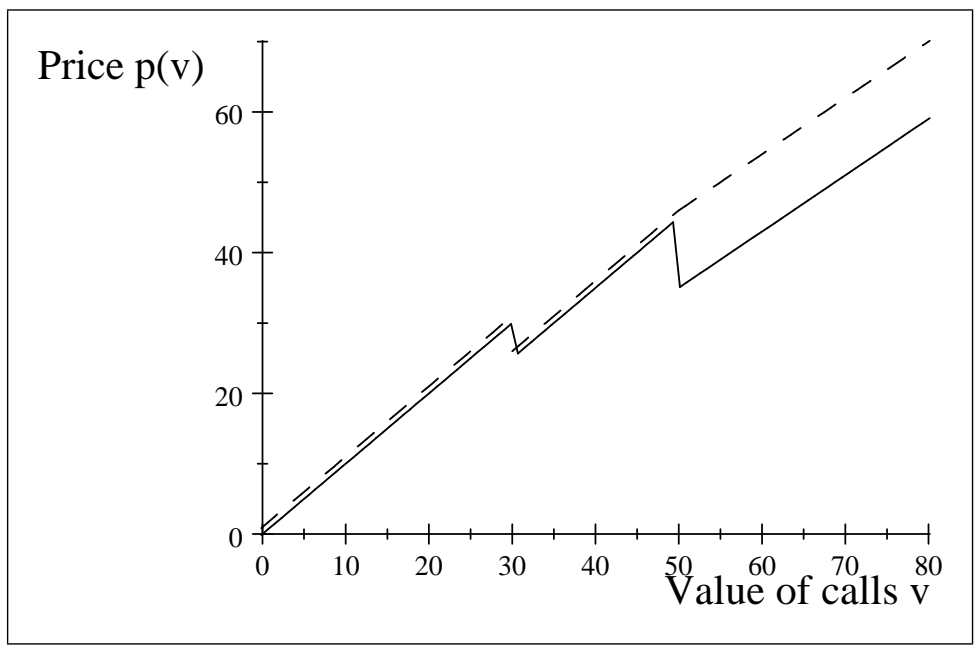

Figure 1: BVD "sawtooth" discount function

Table 1:

Florists' price lists

\begin{tabular}{lcccc} 
& Delivery & \multicolumn{2}{c}{ Items } & Discount \\
\cline { 3 - 4 } & charge & tulip & rose & breakpoint \\
\hline Florist 1 & 10 & 7 & 6 & 50 \\
Florist 2 & 5 & 9 & 3 & 50 \\
\hline
\end{tabular}

Table 2

Optimal transportation solutions

(a) Solution to order for 7 roses \& 3 tulips

\begin{tabular}{rcrrc} 
& Delivery & tulip & rose & Value \\
\hline Florist 1 & 0 & 7 & 1 & 55 \\
Florist 2 & 5 & 0 & 2 & 6 \\
\hline Charge & 5 & 49 & 12 & $\underline{66}$
\end{tabular}

(b) Solution to order for 7 roses

\begin{tabular}{ccrcc} 
& Delivery & tulip & rose & Value \\
\hline Florist 1 & 0 & 7 & 1 & 55 \\
Florist 2 & 0 & 0 & 0 & 0 \\
\hline Charge & 0 & 49 & 6 & $\underline{55}$
\end{tabular}


Table 3:

Comparison of subgradient procedures with and without scaling

\begin{tabular}{cccccccccc} 
& & \multicolumn{3}{c}{ Classical subgradient } & \multicolumn{3}{c}{ r-algorithm } \\
\cline { 2 - 11 } $\begin{array}{c}\text { ORLib } \\
\text { instance }\end{array}$ & $\begin{array}{c}\text { no. } \\
\text { suppliers }\end{array}$ & $\begin{array}{c}\text { no. } \\
\text { items }\end{array}$ & $\begin{array}{c}\text { Scaling } \\
\mathrm{y} / \mathrm{n}\end{array}$ & $\begin{array}{c}\text { no. } \\
\text { branchings }\end{array}$ & $\begin{array}{c}\text { no. } \\
\text { iterations }\end{array}$ & time & $\begin{array}{c}\text { no. } \\
\text { branchings }\end{array}$ & $\begin{array}{c}\text { no. } \\
\text { iterations }\end{array}$ & time \\
\hline Cap 71 & 16 & 50 & $\mathrm{y}$ & 4 & 94 & 3 & 2 & 211 & 6 \\
& & & $\mathrm{n}$ & 21 & 1240 & 12 & 2 & 166 & 5 \\
\hline Cap101 & 25 & 50 & $\mathrm{y}$ & 6 & 43 & 4 & 2 & 201 & 7 \\
& & & $\mathrm{n}$ & 9 & 442 & 8 & 2 & 161 & 7 \\
\hline Cap131 & 50 & 50 & $\mathrm{y}$ & 9 & 115 & 5 & 4 & 250 & 15 \\
& & & $\mathrm{n}$ & 31 & 886 & 27 & 2 & 201 & 13 \\
\hline
\end{tabular}

Table 4:

Effect of discount type and level on execution times (s.)

\begin{tabular}{ccccc} 
Cap131 & \multicolumn{4}{c}{ Timings (s.) } \\
\cline { 2 - 5 } derived & \multicolumn{2}{c}{ Classical } & \multicolumn{2}{c}{ r-algorithm } \\
\cline { 2 - 5 } instance & Unscaled & Scaled & Unscaled & Scaled \\
\hline A0+B0 & 1.8 & 1.4 & 2 & 3 \\
A25 & 191 & 62 & 133 & 147 \\
A40 & 548 & 325 & 545 & 666 \\
A50 & 1413 & 755 & 2492 & 2167 \\
A60 & 1702 & 958 & 2231 & 2246 \\
A75 & 1082 & 316 & 1037 & 645 \\
A100 & 275 & 36 & 110 & 151 \\
B10 & 3 & 3 & 4 & 3 \\
B15 & 6 & 2 & 4 & 3 \\
B20 & 13 & 5 & 4 & 3 \\
A25+B10 & 126 & 36 & 90 & 87 \\
A50+B10 & 106 & 44 & 43 & 46 \\
A75+B10 & 71 & 26 & 15 & 20 \\
A100+B10 & 50 & 6 & 7 & 7 \\
\hline
\end{tabular}


Table 5:

Exact and approximate solution times for "Uniform-123" instances

\begin{tabular}{ccccccc} 
Uniform- $123+$ & $\%$ & Range & \multicolumn{2}{c}{ Classic subgradient } & \multicolumn{2}{c}{ r-algorithm } \\
\cline { 4 - 7 } discount type & Accuracy & factor $D$ & Time s. & Value $Z_{U}$ & Time s. & Value $Z_{U}$ \\
\hline A0+B0 & exact & & 957 & $71342^{*}$ & 1379 & $71342^{*}$ \\
$\vdots$ & $\vdots$ & 2 & 2137 & $\vdots$ & 1557 & $\vdots$ \\
$\vdots$ & $\vdots$ & 1000 & 7065 & $\vdots$ & 1612 & $\vdots$ \\
\hline A0+B0 & $1 \%$ & & 383 & 72034 & 565 & $71342^{*}$ \\
$\vdots$ & $2 \%$ & & 147 & 72606 & 314 & 72080 \\
$\vdots$ & $3 \%$ & & 61 & 73377 & 78 & 73422 \\
$\vdots$ & $\vdots$ & 10 & 938 & 71342 & 403 & 72681 \\
\hline A30 & $3 \%$ & & 293 & 71716 & 1596 & 71344 \\
A50 & $3 \%$ & & 903 & 68815 & 2836 & 68902 \\
$\vdots$ & $5 \%$ & & 292 & 70396 & 411 & 69476 \\
B20 & $3 \%$ & & 92 & 72282 & 154 & 71704 \\
B50 & $\vdots$ & & 99 & 71086 & 5442 & 72375 \\
\hline
\end{tabular}

